\title{
An organism arises from every nucleus
}

\section{Nurullah Keklikoglu}

Istanbul University, Faculty of Dentistry, Department of Histology and Embryology, Istanbul, Turkey.

\begin{abstract}
The fact that, cloning using somatic cell nuclear transfer (SCNT) method has been performed, opened new horizons for cloning, and changed the way of our understanding and approach to cell and nucleus. The progress in cloning technology, brought the anticipation of the ability to clone an organism from each somatic cell nucleus. Therefore, the 'Cell Theory' is about to take the additional statement as "An organism arises from every nucleus". The development of gene targeting procedures which can be applied with SCNT, showed us that it may be possible to obtain different versions of the original genetic constitution of a cell. Because of this opportunity which is provided by SCNT, in reproductive cloning, it would be possible to clone enhanced organisms which can adapt to different environmental conditions and survive. Furthermore, regaining the genetic characteristics of ancestors or reverse herediter variations would be possible. On the other hand, in therapeutic cloning, more precise and easily obtainable alternatives for cell replacement therapy could be presented. However, while producing healthier or different organisms from a nucleus, it is hard to foresee the side effects influencing natural processes in long term is rather difficult.
\end{abstract}

Key-words: cloning, SCNT, nuclear transfer, NT, stem cell, cell theory

According to 'Cell Theory', all eukaryotic organisms consists of cells and these cells are the smallest independent units of life. This theory first suggested by Botanist Matthias Schleiden (1838) and zoolog Theodore Schwann (1839). Afterwards, the famous aphorism of Rudolf Virchow (1855) 'all cells arise only from pre-existing cells' (Omnis cellula e cellula) and the addition of Eduard Strasburger (1897) 'all nuclei arise only from pre-existing nuclei' (Omnis nucleus e nucleo) has shaped the theory into its known and generally accepted form $[1,2]$. The progress in cloning technology, brought the anticipation of the ability to clone an organism from each somatic cell nucleus. As the technology of cloning an individual from an adult nucleus which is completed its differentiation is further developed, cell theory is about to take the additional statement as "An organism arises from every nucleus". Furthermore, the development in cloning technology has shown that it may be possible to clone organisms which contain different ver-

Correspondence: N. Keklikoglu, Istanbul Universitesi, Dishekimligi Fakultesi, Histoloji ve Embriyoloji BD, Capa, Istanbul, Turkey; tel.: (+90212) 4142020-30221,

e-mail: nkeklik@istanbul.edu.tr sions of original genetic constitution from every nucleus.

Cloning means generating a series of living beings which have identical genetic structure, and nowadays it is widely performed by nuclear transfer (NT). NT is a method of cloning which involves the procedure of removing the genetic material containing nucleus (karyoplast) of a donor cell and transferring it into an enucleated oocyte or zygote (cytoplast). Metaphase II oocyte (M-II oocyte) is the most widely used cytoplast. In cloning by NT, cultured donor cells in $\mathrm{G}_{0} / \mathrm{G}_{1}$ phase are placed in perivitelline space of enucleated M-II oocyte and they are subjected to fusion and stimulated for mitosis using electrical current [3-5]. Cloning which is performed by transfer of the nucleus of undifferentiated embryonic cell (blastomer) is called embryonic cell nuclear transfer (ECNT) and the method using the transfer of the somatic cell nucleus of an adult is called somatic cell nuclear transfer (SCNT). In terms of success rate, evaluation and comparision of cloning studies is open to discussion. It is claimed that there is no difference between adult and fetal cells in terms of NT success rate [6], however the success rate of reproductive cloning performed using embryonic stem cells are found to be 5-10 times better than using somatic cells of donors [7]. The progress of 
embryo cloning from embryonic, fetal or adult sourced cultured cells shows that potential applications of NT will have a wide range of implementation [8]. As a result of improvements of gene targeting procedure on cultured cells which are used as nucleus donors in cloning method, potential applications such as production of organs for transplantation, basic research in control of gene expression and functions and the role of genetic and epigenetic alterations in diseases and normal growth, may be available $[9,10]$.

According to their purposes, cloning studies can be categorized in two groups: therapeutic cloning and reproductive cloning. In therapeutic cloning, pluripotent stem cells are obtained from the embryo created by cloning of an adult cell for treatment purposes. The aim of this method can be described as cell-replacement therapy [11]. In reproductive cloning studies, lambs were cloned using NT application from a cell passage which is cultured from cells obtained from sheep embryo. Therefore, obtaining a mammalian offspring, an opportunity arises to analyse and modify gene functions [12]. After this advancement, a lamb was cloned from adult mammary gland cell by SCNT. The birth of a lamb from a somatic cell of an adult, showed that there is no irreversible modification of genetic material in differantiated cells [13]. However, because of cell reprogramming in reproductive cloning, it is claimed that clone becomes a different individual than donor [14].

The fact that mammals were cloned from differentiated donor cells by SCNT, showed that even the cells completed their differentiation have the entire genetic information (nuclear totipotency) to create an entirely grown organism [10], and disproved the belief "development is an irreversible process" which is an old dogma [15]. In differentiated somatic cells, DNA metilation model, which is an epigenetic modification, mediating important aspects of genom functions, is usually stable. However, metilation model of nucleus is reprogrammed in cloning [16]. Nuclear reprogramming induces epigenetic profile changes and confers pluripotency on specialized somatic nuclei. Hybrid cells obtained by the in vitro fusion of adult somatic cell nucleus with embryonic stem cells, can be reprogrammed [17].

Gene targeting procedures allowing some operations on nucleus which will be cloned, are still being improved. When SCNT and gene targeting precedures are applied together, it will provide a very wide range field of study. Altough this situation may cause some expected concerns, it carreis new and fundementally important hopes.

Due to SCNT, it will be possible to clone organisms which can easily adapt to changing environmental conditions or create new species that can survive under different environmental conditions. It is known that the species which can not adapt to natural conditions will be extinct, and variant species which fit the conditions can reproduce and survive. These mutations are not only crucially important for existence of species but also they may be essential for biologic functions of an individual. However, after mutation process, variant species may lose their not required but beneficial herediter characteristics.

SCNT allows us to observe the development of offspring, thus SCNT present opportunities such as research and control on the differentiation, dedifferentiation and transdifferentiation of cells during these processes [18]. It may be possible to overcome the problem of cell differentiation stability, because of nuclear reprogramming feature of SCNT. Genes which are silent in differentiated form of cell, become active, however genes peculiar to differentiated form, renders ineffective during reprogramming process [19]. This provides the ability to detect former healthier conditions and affect and reverse the mutations in the long run. It is possible for nucleus to gain the genetic characteristics of its ancestors under former environmental conditions, provided by a minimalized microenvironment.

The technology allowing us to analyse genetic material and operate on it, is still developing in terms of diagnosis and devices. Three-dimensional (3D) chromatine nanostructure and the topologic analysis of cell nucleus can be performed with high resolution microscopes, and using these devices gene localizations can be defined much more precisely and accurately [20]. Methods to group genetic materials according to their characteristics are also being developed. It is possible to research chromosomes and chromatine in nucleolus using immunoelectron microscope (IEM) method [20,21]. Template (old) and new synthesized DNA strands can be labelled separately [22]. As these methods further develop, detection of determination and tracking of maternal and paternal genome is getting easier. Researchers provide a very useful approach in order to study of alterations of peculiar to maternal genom in mammalian embryos by placing an additional female genom into a zygote [23].

Since biotechnological applications enlighten us on genes functional process, chance of gene tracking and operation increases. If the maternal and paternal chromosomes of a somatic cells are separately transcripted and this genetic material could be cloned by SCNT, it may be possible to clone ancestoral genes, including even the ones from several generations ago.

SCNT would present more relaible, precise and easly obtainable alternatives for cell replacement therapy in therapeutic cloning area. There is no doubt, that the methods which allow us to use patient's own cells directly, would be more ethical. However, developments of cloning technology promises that ethical concern would be satisfied without even needing this approach. 
It may be possible to reverse the differentiation process of a somatic cell. It can be achieved by dysfunctionalization or preventing the conditions needed to function without exposing it to pathologic factors in vivo. It can be also achieved using hybridization technology. Under these conditions, the cell will turn into pluripotent stem cell. If this occurs, it could be considered that every cell can potentially be a stem cell.

There are limited application areas according to types and differentiation potential of stem cells. Stem cells or progenitor cells can actively reproduce in culture [24]. The most important aim in this area is to detect and reproduce the stem cells which have superior characteristics and potential. Some stem cells may have superior characteristics compared to others. For example, although, stem cells in crypts of small intestinal mucosa of mice, divided approximately one thousand times in their entire lifetime, there is a little reduction in their proliferative potential and they are exposed to carcinogenic mutations rarely, and it is claimed that the genom of cells in this example are overprotected [22]. Pluripotent embryonic stem cells in inner cell mass of embryo show excessive selfrenewal in culture and preserve their differentiation ability to all cell types. These characteristics make the embryonic stem cells a good candidate for cell replacement therapy [11]. It is required to find the most viable stem cell line in order to provide differentiation to specific cell types (neurons, muscle cells, etc) of pluripotent stem cells. In order to create new cell lines, preimplantation blastocysts should be destroyed [25]. However, it brings ethical discussions. Therefore, finding the way to transform adult stem cells into pluripotent cells is a much more acceptable alternative [11].

Hybridization may be a solution to obtain qualified stem cells. Embryonic stem (ES) cells maintain nuclear reprogramming activity if they are subjected to cell fusion with adult somatic cells. Reprogrammed somatic nuclei resemble ES cell nuclei in pluripotential competence. The somatic-derived Nonog gene which is responsible from maintaining continuity of pluripotence is also reactivated in the reprogrammed nuclei in hybrid cells and also in cloned blastocysts. A new technological advancement providing the elimination of ES cell originated chromosomes of hybrid cells, may allow the production of pluripotent stem cells without needing therapeutic cloning [17]. The ideal sources for research of pluripotent stem cells obtained by cell fusion are adult mesenchymal stem and progenitor cells. Mesenchymal stem cells can be proliferated as differentiated cells and these can also differentiate to some series of mesenchymal tissues such as bone, cartilage, adipose, tendon, muscle and bone marrow stroma [26]. Mesodermal progenitor cells proliferates without showing an obvious aging and because they differentiate not only to mesenchymal cells but also to visceral mesoderm, these cells may be a stem cell source for treatment of genetic and degenerative disorders affecting mesodermal originated cells [27].

The most practical but difficult at the beginning way to solve the problem, "which cells should be used in which tissue", is obtaining a qualified totipotent ES cell and its passages which can be applied to all humans and tissues. Alternatively, at least, for each germ layer (ecto-, endo-, mesoderm), a different cell passage should be developed. If cells which can be used regularly can not be obtained for cell replacement therapy, a vast genetic reserve will be needed. Not only being a reserve, DNA bank is an important research material for human epidemiological studies [28]. Nucleus bank also provides a biological population registery for observation of development of species and genetic research. This way, a wide range somatic nuclear reserve is established in order to be used in tissue engineering in upcoming years.

SCNT can also be used to produce gametes. There are some different troubles of obtaining a gamet from a somatic cell, when it is needed for reproductive health. The procedure of producing functional male or female artificial gamete from an adult somatic cell using NT is called as haploidization. Haplodization is the process of obtaining haploid cells by removing chromosomes from a diploid cell. Attemps has not been fully succeeded yet, since there is important alterations in spindle construction and chromosomal segregation [29]. It is shown that, with a designed culture system, male germ cells in vertebrate can complete their mitosis and meiosis processes until functional sperm is created [30]. Obtaining a gamete from stem cells created by SCNT, using specific culture conditions, will be possible after overcoming the difficulties in mitotic-meiotic cell division, genetic-epigenetic arrangements and reconstruction of cells [29].

In fact, in haplodization what should be done is may be reversing zygote creation process in principle. Obtaining blastomers which are closest cells to zygote by SCNT, can smooth the way for haplodization.

The difference between male and female cells is an important detail for cell-replacement therapy. In males, testosterone expressed by testes differentiated trough mammalian Y-linked gene Sry affects organs and causes masculine patterns of growth. Therefore, some XX and $\mathrm{XY}$ tissues, become sexually dimorphic althought, they develop in a similar endocrine environment [31]. While $\mathrm{X}$ chromosome is considered to be the subsequent of ancestral genes from evolutionary times, counterpart chromosome $\mathrm{Y}$ is degenerated owing to its lack of recombination [32]. Therefore, cells of a male not carrying Y chromosome may be considered healthier in some ways.

Being already responsible from reproduction, usage of gametes in cloning studies may prove to be 
advantageus. Genotype-phenotype studies have shown that, only one phenotype, sex, has perfection and binary correlation with genotype [28]. Techniques such as multiple-color primed in situ labelling (multi-PRINS), multiple-fluorescence in situ hybridization (multiFISH) and comparative genomic hybridization (CGH) provide identification of molecularly differentiated sex chromosomes and DNA composition of sex chromosomes [33,34]. It possible to create new combinations defining chromosomal details of meiotic division and utilising separation of chromosomes and their transcription. This depends on the condition of elucidation of DNA reconstruction principles. When a second $\mathrm{X}$ chromosome is created in a somatic cell which is forced to meiosis or $\mathrm{Y}$ chromosomes of which is removed, this hypothesis may come true.

Sex chromosomes are derived from ordinary autosomes [32]. For example, human spermiums derived from spermatogoniums which have $44 \mathrm{~A}+\mathrm{XY}$ chromosomal combination. They become $22 \mathrm{~A}+\mathrm{X}$ and $22 \mathrm{~A}+\mathrm{Y}$ combinated chromosomes by meiotic division during spermatogenesis. This incidence shows that $\mathrm{X}$ and $\mathrm{Y}$ chromosomes can be divided from a healty cell in a natural way. If two separate spermatid bearing $22 \mathrm{~A}+\mathrm{X}$ chromosome which belong to a male can be used in cloning technology, it may be possible to clone opposite sex (a female) from a male. In this case, it may be possible to obtain cells which does not bearing a Y chromosome from a male to a female for currently advancing human tissue engineering studies.

Researchers have created XX-XY chimeras and have shown that, female cells are able to functionally exist in male organs. It has been observed that, testicular eggs (XX germ cells) produced in seminiferous tubules of $\mathrm{XX}-\mathrm{XY}$ chimeric testes resume oogenesis, have an eggspecific structure and zona pellucida and are able to fuse with sperms. Determining that, testicular eggs undergo maternal imprinting despite testicular environment, it is shown that, genomic imprinting does not necessarily always follow environmental conditions which lets germ cells survive [35]. These data shows that male cells may not lose their maternal characteristic in endocrinal environment. Although, it is not possible to create a male cell from a female cell due to absence of Y chromosome, or only way to achieve it is to introduce a Y chromosome to the female cell, it is possible to clone a female cell from a male cell.

These hypotheses carry vital importance especially for cell-replacement therapy applications on human. However, these studies can be possible and useful only establishing a general consensus following legal arrangements and ethical discussions. It is also obvious, that much more detailed information and technology is needed to perform these studies.

It seems that providing ideal health conditions and supporting reproduction of mammalian species will be possible by their own cells. The statement "An organism arises from every nucleus" is not only describing the current status, like accepted cell theory statements, it also points out the starting point of which may be possible in near future. Cloning a different or a healthier organism from a nucleus may be possible, however it is unclear and rather difficult to foresee its long term effects on natural processes.

\section{References}

[ 1] Baluska F, Volkmann D, Barlow PW. Eukaryotic cells and their cell bodies: Cell Theory revised. Ann Bot (Lond). 2004;94:9-32.

[ 2] Breathnach CS. Rudolf Virchow (1821-1902) and Die Cellularpathologie (1858). J Ir Coll Physicians Surg. 2002;31:4346.

[ 3] Li Z, Rezaei Sabet M, Zhou Q, et al. Developmental capacity of ferret embryos by nuclear transfer using G0/G1-phase fetal fibroblasts. Biol Reprod. 2003;68:2297-2303.

[ 4] Pralong D, Mrozik K, Occhiodoro F, et al. A novel method for somatic cell nuclear transfer to mouse embryonic stem cells. Cloning Stem Cells. 2005;7:265-271.

[ 5] Hosaka K, Sato Y, Okamoto N, Kazami A, Sato K. Development of reconstituted embryos derived from somatic cell nuclei in the rabbit. Hum Cell. 2004;17:29-32.

[6] Yin XJ, Lee HS, Lee YH, et al. Cats cloned from fetal and adult somatic cells by nuclear transfer. Reproduction. 2005;129:245-249.

[ 7] Sung LY, Gao S, Shen H, et al. Differentiated cells are more efficient than adult stem cells for cloning by somatic cell nuclear transfer. Nat Genet. 2006;38:1323-1328.

[ 8] Wolf E, Zakhartchenko V, Brem G. Nuclear transfer in mammals: recent developments and future perspectives. J Biotechnol. 1998;65:99-110.

[ 9] Paterson L, DeSousa P, Ritchie W, King T, Wilmut I. Application of reproductive biotechnology in animals: implications and potentials. Applications of reproductive cloning. Anim Reprod Sci. 2003;79:137-143.

[10] Meissner A, Jaenisch R. Mammalian nuclear transfer. Dev Dyn. 2006;235:2460-2469.

[11] Kues WA, Carnwath JW, Niemann H. From fibroblasts and stem cells: implications for cell therapies and somatic cloning. Reprod Fertil Dev. 2005;17:125-134.

[12] Campbell KH, McWhir J, Ritchie WA, Wilmut I. Sheep cloned by nuclear transfer from a cultured cell line. Nature. 1996;380:64-66.

[13] Wilmut I, Schnieke AE, McWhir J, Kind AJ, Campbell KH. Viable offspring derived from fetal and adult mammalian cells. Nature. 1997;385:810-813.

[14] Oakley J. Reproductive cloning and arguments from potential. Monash Bioeth Rev 2006;25:42-47.

[15] Hochedlinger K, Jaenisch R. Nuclear reprogramming and pluripotency. Nature. 2006;441:1061-1067.

[16] Reik W, Dean W, Walter J. Epigenetic reprogramming in mammalian development. Science. 2001;293:1089-1093.

[17] Tada T. Toti-/pluripotential stem cells and epigenetic modifications. Neurodegener Dis. 2006;3:32-37.

[18] Trounson AO. Future and applications of cloning. Methods Mol Biol. 2006;348:319-332.

[19] Simonsson S, Gurdon JB. Changing cell fate by nuclear reprogramming. Cell Cycle. 2005;4:513-515.

[20] Esa A, Edelmann P, Kreth G, et al. Three-dimensional spectral precision distance microscopy of chromatin nanostructures after triple-colour DNA labelling: a study of the BCR 
region on chromosome 22 and the Philadelphia chromosome. J Microsc. 2000;199:96-105.

[21] Ghosh S, Paweletz N. Detection of intranucleolar chromatin using an ultrastructural immunolabelling technique. Cell Biol Int. 1998;22:609-614.

[22] Potten CS, Owen G, Booth D. Intestinal stem cells protect their genome by selective segregation of template DNA strands. J Cell Sci. 2002;115:2381-2388.

[23] Plusa B, Grabarek JB, Karasiewicz J, Modlinski JA. Meiotic maternal chromosomes introduced to the late mouse zygote are recruited to later embryonic divisions. Mol Reprod Dev. 2005;70:429-437.

[24] Moore KA, Ema H, Lemischka IR. In vitro maintenance of highly purified, transplantable hematopoietic stem cells. Blood. 1997;89:4337-4347.

[25] Fischbach GD, Fischbach RL. Stem cells: science, policy, and ethics. J Clin Invest. 2004;114:1364-1370.

[26] Pittenger MF, Mackay AM, Beck SC, et al. Multilineage potential of adult human mesenchymal stem cells. Science. 1999;284:143-147.

[27] Reyes M, Lund T, Lenvik T, Aguiar D, Koodie L, Verfaillie CM. Purification and ex vivo expansion of postnatal human marrow mesodermal progenitor cells. Blood. 2001;98:26152625.

[28] Huang S, Chen XH, Day IN. Combination of 768-well microplate array diagonal gel electrophoresis with duplex
PCR of X and Y chromosome markers for quality control of epidemiological DNA banks. Electrophoresis. 2006;27:30383041.

[29] Nagy ZP, Chang CC. Artificial gametes. Theriogenology. 2007;67:99-104.

[30] Sakai N. Transmeiotic differentiation of zebrafish germ cells into functional sperm in culture. Development. 2002;129:3359-3365.

[31] Arnold AP, Burgoyne PS. Are XX and XY brain cells intrinsically different? Trends Endocrinol Metab. 2004;15:6-11.

[32] Bachtrog D. A dynamic view of sex chromosome evolution. Curr Opin Genet Dev. 2006;16:578-585.

[33] Traut W, Eickhof U, Schorch JC. Identification and analysis of sex chromosomes by comparative genomic hybridization (CGH). Methods Cell Sci. 2001;23:155-161.

[34] Gadji M, Krabchi K, Drouin R. Simultaneous identification of chromosomes $18, \mathrm{X}$ and $\mathrm{Y}$ in uncultured amniocytes by using multi-primed in situ labelling technique. Clin Genet. 2005;68:15-22.

[35] Isotani A, Nakanishi T, Kobayashi S, et al. Genomic imprinting of XX spermatogonia and XX oocytes recovered from $\mathrm{XX}<->\mathrm{XY}$ chimeric testes. Proc Natl Acad Sci USA. 2005;102:4039-4044.

Submitted: 5 January 2009 Accepted after reviews: 23 April 2009 